\section{Condutas de risco à saúde e indicadores de estresse psicossocial em adolescentes estudantes do Ensino Médio}

\author{
Health risk behaviors and psychosocial distress \\ indicators in high school students
}

\author{
1 Faculdade de Odontologia, \\ Universidade de Pernambuco, \\ Recife, Brasil. \\ 2 Escola Superior \\ de Educação Física, \\ Universidade de Pernambuco, \\ Recife, Brasil. \\ Correspondência \\ P. D. Carvalho \\ Programa de Pós-graduação \\ em Hebiatria, Faculdade de \\ Odontologia, Universidade de \\ Pernambuco. \\ Rua Jonathas de Vasconcelos \\ 427, apto. 803, Recife, PE \\ 51021-140, Brasil. \\ prifermeira@hotmail.com
}

\begin{abstract}
The purpose of this article was to examine the association between health risk behaviors (tobacco, alcohol, and drug use) and psychosocial distress indicators among high school students. The sample consisted of 4,210 adolescent students from public schools in Pernambuco State, Brazil. The Global School-based Student Health Survey was used to collect personal (demographic and socioeconomic) and behavioral data and to obtain measures of psychosocial distress indicators (outcome variables). Prevalence rates were observed for sadness, loneliness, suicidal ideation, sleeplessness due to worries, and suicidal planning. Self-reported prevalence rates for tobacco, alcohol, and drug use were $7.7 \%, 30.3 \%$, and 6.9\%. Psychosocial distress was more prevalent among girls, while health risk behaviors were more common among boys. The study concludes that drug use is directly associated with suicidal ideation and planning, and that among girls, alcohol consumption was associated with psychosocial distress.
\end{abstract}

Psychological Stress; Suicide; Adolescent; Health Behavior
Priscila Diniz de Carvalho 1

Mauro Virgilio Gomes de Barros 2

Rodrigo Antunes Lima 2

Carla Menêses Santos 2

Edilânea Nunes Mélo 2

\section{Introdução}

Condutas de risco à saúde que são incorporadas ao estilo de vida durante a adolescência tendem a ser mais estáveis durante a vida adulta e, portanto, mais difíceis de modificar ${ }^{1,2}$. Além disso, fatores comportamentais como a inatividade física, o tabagismo e o abuso de álcool estão associados ao maior risco de incidência de doenças crônicas não transmissíveis, como as doenças cardiovasculares 3,4 . Comportamentos sexuais de risco, por sua vez, podem estar associados à ocorrência de gravidez precoce ou indesejada 5 , violência interpessoal 6 e sintomas de depressão 7 .

Estudos internacionais identificaram redução nas prevalências de exposição a essas condutas de risco em populações jovens 8,9, mas as mesmas continuam sendo altas tanto em países economicamente desenvolvidos 8,9 quanto no Brasil 10,11,12. O uso na vida de tabaco, bebidas alcoólicas e drogas ilícitas foi relatado, respectivamente, por $24,2 \%, 71,4 \%$ e $8,7 \%$ dos estudantes de 26 capitais brasileiras e do Distrito Federal 11. Esse mesmo levantamento revelou também que aproximadamente 1 em cada 5 estudantes referiu já ter ficado embriagado pelo menos uma vez na vida. A exposição a essas condutas, além de constituir risco direto à saúde, pode representar a porta de entrada para adoção de outros comportamentos prejudiciais à saúde dos jovens 13,14. 
Há evidências também de que a exposição a condutas de risco como o tabagismo, o consumo de bebidas alcoólicas e o uso de drogas está associado ao estresse psicossocial em adultos 15. Mas essa inter-relação permanece pouco explorada em estudos com adolescentes 16,17,18, e até onde se tem conhecimento nenhum trabalho com abrangência estadual foi conduzido no Brasil.

O suicídio passou a ser encarado como um dos principais problemas de saúde em populações jovens, situando-se como as três principais causas de morte nos Estados Unidos, Suíça e Canadá entre pessoas na faixa etária de 15-24 anos 16. A exposição ao tabagismo, ao consumo de bebidas alcoólicas e ao uso de drogas na adolescência pode ser fator associado ao estresse psicossocial, e a análise desta inter-relação poderá oferecer subsídios ao planejamento de intervenções e de outras investigações. É importante também porque grande proporção de adolescentes refere estresse psicossocial, tais como sentimento de solidão, dificuldade para dormir devido à preocupação, sentimento de tristeza, pensamento de suicídio e planos de suicídio 16,17,18.

Além da relativa escassez de trabalhos focalizando a associação entre condutas de risco à saúde e indicadores de estresse psicossocial em adolescentes brasileiros, outros dois elementos justificam a realização do estudo: (1) a necessidade de confirmar se a associação entre condutas de risco e estresse psicossocial, observada em adultos e adolescentes de outros contextos socioculturais, também se verifica em adolescentes brasileiros; e (2) os estudos disponíveis analisaram a associação entre condutas de risco à saúde e um único ou poucos indicadores de estresse psicossocial, usualmente sem controle de variáveis de confusão. Assim, o objetivo do presente estudo foi analisar a associação entre condutas de risco à saúde (tabagismo, consumo de bebidas alcoólicas e uso de drogas) e indicadores de estresse psicossocial em adolescentes estudantes do Ensino Médio.

\section{Métodos}

Este estudo epidemiológico transversal foi desenvolvido como parte do projeto de pesquisa denominado Estilos de Vida e Comportamentos de Risco à Saúde em Estudantes do Ensino Médio no Estado de Pernambuco. O protocolo do estudo foi submetido ao Comitê de Ética em Pesquisa com Seres Humanos do Hospital Agamenon Magalhães, na cidade do Recife, Pernambuco, em atendimento à indicação do Sistema Nacional de Ética na Pesquisa com Seres Humanos, tendo sido aprovado em julho de 2005.
A população-alvo foi limitada aos adolescentes (14-19 anos), estudantes da rede pública estadual de Ensino Médio do Estado de Pernambuco. Considerando-se todas as dependências administrativas (federal, estadual, municipal e privada), os dados fornecidos pela Secretaria de Educação do Estado indicavam que, em 2005, os sujeitos matriculados na rede pública estadual representavam cerca de $80 \%$ do total de estudantes do Ensino Médio em Pernambuco. O dimensionamento da amostra foi efetuado de forma a atender os diversos objetivos do projeto, que incluíam a avaliação da exposição a dez fatores de risco à saúde, dentre os quais os indicadores de estresse psicossocial.

Para o cálculo do tamanho da amostra foram utilizados os seguintes critérios: população estimada em 353 mil sujeitos; intervalo de 95\% de confiança (IC95\%); erro amostral de 3 pontos percentuais; prevalência estimada em $50 \%$; e efeito de delineamento amostral estabelecido em quatro vezes o tamanho mínimo da amostra. Isso representaria uma amostra mínima com 4.217 sujeitos.

Procurou-se garantir que a amostra selecionada representasse a população-alvo quanto à distribuição conforme região geográfica, porte das escolas e período de matrícula dos estudantes (diurno e noturno). A distribuição regional foi observada pelo número de escolas existentes em cada uma das 17 GEREs (Gerências Regionais de Ensino). A fim de subsidiar o planejamento amostral, as escolas foram classificadas em três categorias: pequeno porte (menos de 200 alunos); médio porte (200 a 499); e grande porte (500 alunos ou mais). Alunos matriculados nos períodos da manhã e da tarde foram agrupados numa única categoria (estudantes do período diurno).

Para seleção da amostra requerida, recorreuse a um procedimento de amostragem aleatória estratificada em dois estágios, sendo que a "escola" e a "turma" representaram as unidades amostrais, respectivamente, no primeiro e no segundo estágios. Todas as escolas da rede pública estadual em Pernambuco foram consideradas elegíveis para inclusão no estudo. No primeiro estágio, adotou-se como critério de estratificação para realização do sorteio a densidade de escolas em cada microrregião do estado (GEREs) por porte. No segundo, considerou-se a densidade de turmas nas escolas sorteadas por período (diurno e noturno) como critério para sorteio das turmas nas quais os questionários seriam aplicados. Todos os estudantes das turmas sorteadas foram convidados a participar do estudo. Os sorteios foram realizados mediante geração de número aleatórios usando-se o programa Epi Info, versão 
6.04d (Centers for Disease Control and Prevention, Atlanta, Estados Unidos).

A coleta dos dados foi realizada no período de abril a outubro de 2006. A pesquisa (aplicação do questionário) foi efetuada por seis mestrandos do Programa de Pós-graduação em Hebiatria da Universidade de Pernambuco, previamente treinados para padronização dos procedimentos de coleta de dados. O treinamento desses aplicadores abrangeu um curso ministrado pelos pesquisadores aos estudantes de pós-graduação, e a participação na coleta de dados do estudo piloto como preparação para o trabalho de campo.

O questionário autoadministrado foi aplicado em sala de aula sem a presença dos professores, sendo que todos os estudantes presentes foram convidados a participar, independentemente da idade. Além disso, o questionário não continha qualquer tipo de identificação pessoal (nome, matrícula). Os estudantes foram continuamente assistidos pelos aplicadores (sempre dois por turma) para que pudessem esclarecer dúvidas e auxiliar no preenchimento das informações. A participação dos sujeitos foi voluntária e anônima, adotando-se a utilização de termo negativo de consentimento (parental passive consent form).

O questionário utilizado para obtenção de dados foi uma versão traduzida, autoadministrada e previamente testada do Global School-based Student Health Survey (GSHS), proposto pela Organização Mundial da Saúde (OMS; http://www. who.int/chp/gshs/en). O questionário é composto por dez módulos: características pessoais; consumo de álcool e drogas; hábitos alimentares; higiene; saúde mental (do qual derivaram os indicadores de estresse psicossocial); atividades físicas; comportamentos na escola; comportamento sexual; tabagismo; e violência. A utilização de questionário em estudos epidemiológicos com foco na análise de indicadores de estresse psicossocial é um procedimento metodológico que vem sendo adotado em investigações congêneres 16,17,18 devido ao baixo custo e facilidade de aplicação.

Previamente ao início da coleta de dados, um estudo piloto foi conduzido a fim de determinar indicadores de reprodutibilidade de medidas e testar a aplicabilidade do instrumento. Os dados para o estudo piloto foram coletados em duas escolas da rede pública municipal de ensino na cidade do Recife, com uma amostra de 138 adolescentes com idades de 14-19 anos (59 moças). Indicadores de reprodutibilidade (consistência de medidas teste-reteste) foram de moderados a altos na maioria dos itens do instrumento, sendo que os coeficientes de concordância (índice kappa) para medidas dos indicadores de estresse psicossocial variaram de 0,60 (qualidade do sono) a 0,93 (pensamento de suicídio). Os índices de reprodutibilidade para as medidas de exposição a condutas de risco à saúde variaram de 0,63 (consumo de bebidas alcoólicas) a 0,93 (uso de drogas).

Cinco indicadores de estresse psicossocial (sentimento de solidão, dificuldade para dormir devido à preocupação, sentimento de tristeza, pensamento de suicídio e planos de suicídio) foram analisados neste estudo e definidos operacionalmente como variáveis dependentes. Sentimento de solidão foi determinado mediante análise das respostas à questão: "Durante os últimos 12 meses, com que frequência você se sentiu sozinho?", sendo que todos os sujeitos que referiram "a maioria das vezes" ou "sempre" foram classificados como casos. Similarmente, dificuldade para dormir devido à preocupação foi identificada pela resposta à pergunta: “ $D u$ rante os últimos 12 meses, com que frequência você esteve tão preocupado com alguma coisa que não conseguiu dormir à noite?", sendo que os estudantes que responderam "a maioria das vezes" ou "sempre" foram classificados como casos. Casos de sentimento de tristeza foram identificados por respostas afirmativas à questão: " $D u$ rante os últimos 12 meses, você se sentiu 'muito triste' ou 'sem esperança' quase todos os dias durante duas semanas ou mais seguidas, a ponto de você ter que parar de fazer suas atividades normais?". Pensamento de suicídio foi identificado pela resposta afirmativa dos sujeitos à pergunta: "Durante os últimos 12 meses você pensou em se suicidar?". Na variável plano de suicídio, os casos foram identificados pela resposta afirmativa dos estudantes à pergunta: "Durante os últimos 12 meses você fez planos para se suicidar?”.

Foram considerados expostos ao consumo de tabaco todos os sujeitos que relataram ter fumado cigarro em pelo menos 1 dos últimos 30 dias (uso atual), independentemente da intensidade de exposição (frequência e quantidade de cigarros consumidos). De modo similar, foram considerados expostos ao consumo de bebidas alcoólicas (uso atual) todos os estudantes que relataram ter utilizado qualquer tipo de bebida alcoólica em pelo menos 1 dos últimos 30 dias. Considerou-se consumo de drogas o relato de uso na vida de loló, cola de sapateiro, lança perfume, maconha, crack, cocaína ou outras drogas, independentemente da quantidade e frequência. Esses três fatores foram considerados nas análises como variáveis independentes.

A idade dos estudantes foi dicotomizada nas seguintes faixas etárias: 14-16 e 17-19 anos. A cor da pele/etnia foi autorreferida considerando-se 
seis possíveis respostas (branca, preta, parda, indígena, amarela e outra) e, em seguida, agrupada em duas categorias (branca e não branca). A variável "morar com os pais" foi dicotômica (sim/ não) e somente os estudantes que referiram morar com pai e mãe foram classificados na categoria "sim". O local de residência foi referido em duas categorias (urbano e rural). A escolaridade materna foi referida pelo filho e classificada em três categorias ( $\leq 8$ anos, 9-11 e 12 anos ou mais de estudos). A situação ocupacional do adolescente foi uma variável dicotômica (trabalha/não trabalha), analisada mediante seu engajamento no mercado de trabalho formal ou informal; assim, todos os que relataram trabalhar, independentemente de ter ou não carteira profissional assinada, foram classificados como trabalhadores. O turno em que estudavam foi agrupado em diurno (abrangendo aqueles que estudavam no período matutino e vespertino) e noturno. A série escolar foi codificada como padronizada no sistema educacional. Esses fatores serviram à caracterização da amostra e ajustamento das análises de associação.

Análises estatísticas foram realizadas mediante utilização do programa Stata para Windows, versão 9 (Stata Corp., College Station, Estados Unidos). Análises descritivas foram efetuadas por distribuição de frequências, enquanto que para as análises bivariáveis recorreu-se à aplicação do teste de qui-quadrado.

Análises de associação entre as variáveis independentes e dependentes foram conduzidas mediante a utilização de regressão logística separadamente para cada uma das variáveis dependentes (indicadores de estresse psicossocial). Inicialmente, procederam-se análises brutas e, em seguida, as análises multivariáveis que foram conduzidas a fim de controlar potenciais fatores de confusão, sendo que tanto as análises brutas quanto as ajustadas foram estratificadas por sexo devido à identificação de interação.

As análises de regressão logística múltipla foram efetuadas para cada desfecho separadamente, incluindo-se no modelo a variável independente a ser testada, faixa etária, morar com os pais, turno e os demais fatores comportamentais de risco. A escola com estratificação por porte (pequeno, médio e grande) e a turma com estratificação por turno (diurno e noturno) foram consideradas, respectivamente, as unidades amostrais primária e secundária que definiram o delineamento amostral. As análises levaram em consideração essa estratégia amostral por conglomerado (opção svyset do Stata) para ajuste de todas as estimativas de precisão calculadas, sem recurso à ponderação. $\mathrm{O}$ valor do efeito do delineamento amostral (deff) foi inferior a 1,40 nos rapazes, com variação de 0,64 (dificuldade para dormir devido à preocupação) a 1,39 (plano de suicídio). Para as moças, variou de 0,98 (sentimento de solidão) a 2,25 (tristeza).

\section{Resultados}

Foram visitadas 76 escolas $(11 \%$ do total de escolas estaduais do estado) em 44 municípios, o que representa $23 \%$ de todos os municípios pernambucanos. Entrevistou-se 4.210 estudantes com idades entre 14 e 19 anos (59,8\% do sexo feminino), sendo que dos sujeitos convidados a participar do estudo 83 se recusaram $(1,9 \%$ de recusas). O detalhamento das características demográficas e socioeconômicas dos sujeitos está apresentado na Tabela 1.

Para atingir o tamanho amostral definido $a$ priori $(\mathrm{n}=4.217)$ mediante a utilização de amostragem por conglomerados foram sorteadas 234 turmas de Ensino Médio (os conglomerados). Estimou-se que isso representaria uma amostra final cerca de $10 \%$ superior ao inicialmente previsto, o que seria desejável devido às possíveis perdas e recusas. Na fase de campo (coleta de dados), verificou-se que o número de estudantes com idade correspondente à faixa etária alvo foi inferior ao estimado, próximo a 18 por turma em média. Além das recusas, essa foi a principal razão para o menor número de estudantes na amostra final em relação ao previsto.

A prevalência de exposição ao tabagismo, consumo de bebidas alcoólicas e uso de drogas está apresentada na Figura 1. Observou-se que a proporção de rapazes expostos a tais condutas de risco à saúde é significativamente $(\mathrm{p}<0,01)$ maior que o verificado entre as moças. A prevalência de exposição ao tabagismo e ao uso de drogas entre os rapazes foi praticamente igual.

Ao observarmos a Figura 2, identifica-se facilmente que a prevalência de indicadores de estresse psicossocial foi significativamente $(\mathrm{p}<$ $0,01)$ maior entre as moças. Independentemente do sexo, sentimento de tristeza e planos de suicídio foram, respectivamente, os indicadores com maior e menor prevalência nesta população de adolescentes estudantes.

As análises de regressão logística bruta evidenciaram que entre os rapazes o consumo de álcool e o uso de drogas são fatores diretamente associados ao pensamento em suicídio. O consumo de álcool pelas moças foi um fator comportamental diretamente associado a todos os indicadores de estresse psicossocial analisados no presente estudo. Ainda entre as moças, a variável uso de drogas foi identificada como fator diretamente associado à tristeza, dificuldade pa- 
ra dormir devido à preocupação, pensamentos e planos de suicídio. Detalhamento desses resultados está apresentado na Tabela 2.

Após a realização das análises bivariáveis recorreu-se à utilização de regressão logística múltipla para cada um dos indicadores de estresse psicossocial, com ajustamento para as variáveis idade, morar com os pais, turno e demais variáveis comportamentais que foram incluídas no modelo como possíveis fatores de confusão. Tanto entre os rapazes quanto entre as moças, verificou-se que o consumo de bebidas alcoólicas está associado ao pensamento de suicídio, desfecho que dentre as moças também está associado à exposição ao uso de drogas na vida (Tabela 3).

Dentre os rapazes, observou-se que a exposição ao uso de drogas na vida é fator associado a planos de suicídio. Por sua vez, entre as moças, a exposição ao consumo de bebidas alcoólicas foi identificada com fator associado a todos os indicadores de estresse psicossocial, exceto "dificuldade para dormir devido à preocupação”.

\section{Discussão}

Os resultados deste estudo permitiram evidenciar importantes achados: (1) exposição a consumo de bebidas alcoólicas e uso de drogas na vida estão diretamente associados a indicadores de estresse psicossocial em adolescentes; (2) a exposição ao tabagismo não foi um fator associado a estresse psicossocial; (3) o uso de drogas na vida foi identificado como um fator associado a planos de suicídio entre os rapazes e pensamento em suicídio entre as moças; (4) a exposição ao consumo de bebidas alcoólicas foi um fator asso-

\begin{tabular}{|c|c|c|c|c|c|c|}
\hline \multirow[t]{2}{*}{ Variável } & \multicolumn{2}{|c|}{ Rapazes } & \multicolumn{2}{|c|}{ Moças } & \multicolumn{2}{|c|}{ Todos } \\
\hline & $\%$ & $\mathrm{n}$ & $\%$ & $\mathbf{n}$ & $\%$ & $\mathrm{n}$ \\
\hline \multicolumn{7}{|l|}{ Idade (anos) * } \\
\hline $14-16$ & 35,5 & 599 & 46,4 & 1.167 & 42 & 1.766 \\
\hline $17-19$ & 64,5 & 1.089 & 53,6 & 1.346 & 58 & 2.435 \\
\hline \multicolumn{7}{|c|}{ Cor da pele/Etnia ** } \\
\hline Branco(a) & 24,8 & 417 & 25,5 & 639 & 25,2 & 1.056 \\
\hline Não branco(a) & 75,2 & 1.263 & 74,5 & 1.868 & 74,8 & 3.131 \\
\hline \multicolumn{7}{|c|}{ 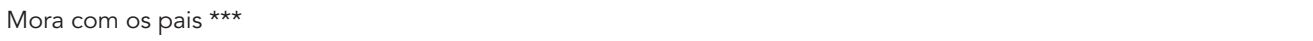 } \\
\hline Sim & 68,1 & 1.131 & 60,6 & 1.512 & 63,6 & 2.643 \\
\hline Não & 31,9 & 529 & 39,4 & 981 & 36,4 & 1.510 \\
\hline \multicolumn{7}{|c|}{ Local de residência \# } \\
\hline Urbana & 78,1 & 1.312 & 79,6 & 1.985 & 79 & 3.297 \\
\hline Rural & 21,9 & 367 & 20,4 & 510 & 21 & 877 \\
\hline \multicolumn{7}{|c|}{ Escolaridade materna (anos de estudo) \#\# } \\
\hline$\leq 8$ & 69,4 & 1.087 & 74,5 & 1.772 & 72,5 & 2.859 \\
\hline $9-11$ & 22,5 & 352 & 20,2 & 481 & 21,1 & 833 \\
\hline$\geq 12$ & 8,1 & 127 & 5,3 & 126 & 6,4 & 253 \\
\hline \multicolumn{7}{|c|}{ Situação ocupacional \#\#\# } \\
\hline Não trabalha & 69,3 & 1.158 & 84,8 & 2.121 & 78,6 & 3.279 \\
\hline Trabalha & 30,7 & 514 & 15,2 & 381 & 21,4 & 895 \\
\hline \multicolumn{7}{|l|}{ Turno § } \\
\hline Diurno & 53,9 & 909 & 60,1 & 1.508 & 57,6 & 2.417 \\
\hline Noturno & 46,1 & 778 & 39,9 & 1.002 & 42,4 & 1.780 \\
\hline \multicolumn{7}{|l|}{ Série §§ } \\
\hline 1으 ano & 46,2 & 779 & 43,8 & 1.101 & 44,8 & 1.880 \\
\hline 2o ano & 30,9 & 522 & 32,6 & 818 & 31,9 & 1.340 \\
\hline 3o ano & 22,9 & 386 & 23,6 & 593 & 23,3 & 979 \\
\hline
\end{tabular}

Dados perdidos: ${ }^{\star} n=9 ;{ }^{* \star} n=23 ;{ }^{* \star *} n=57 ; \# n=36 ; \# n=265 ; \# \# n=36 ; \S n=13 ; \S \S n=11$. 
Figura 1

Prevalência de exposição ao tabagismo, consumo de bebidas alcoólicas e uso de drogas em adolescentes estudantes do Ensino Médio por sexo. Estado de Pernambuco, Brasil, 2006.

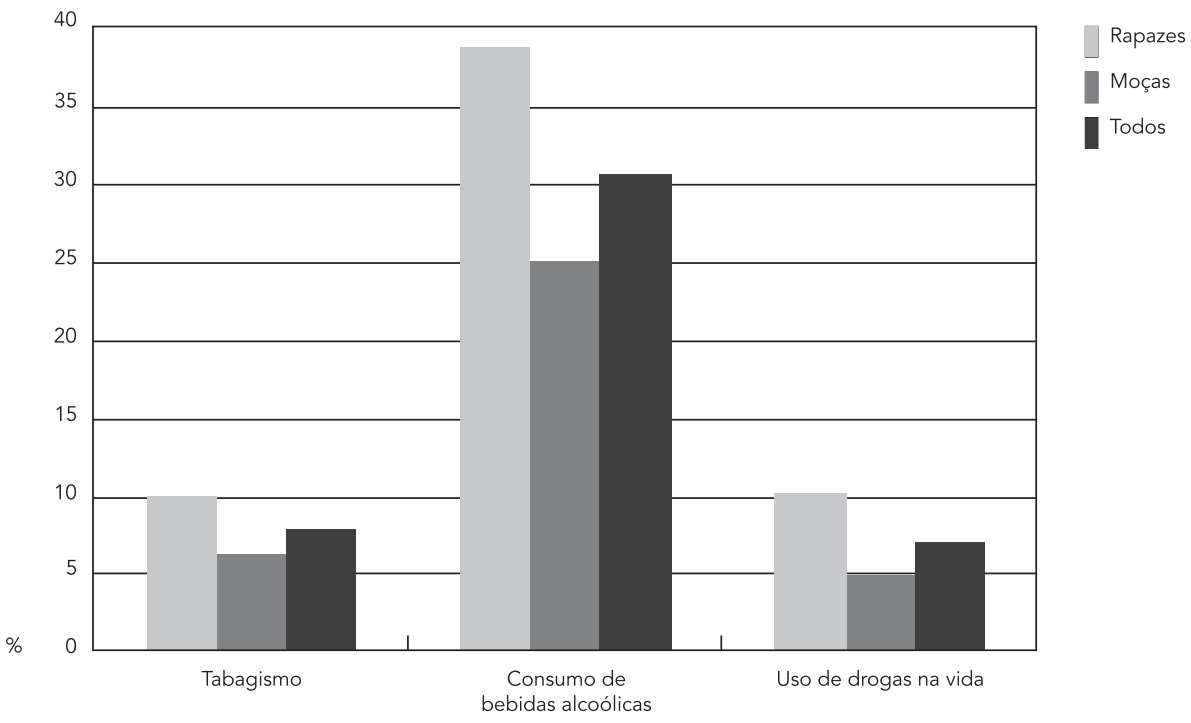

Figura 2

Prevalência de indicadores de estresse psicossocial em adolescentes estudantes do Ensino Médio por sexo. Estado de Pernambuco, Brasil, 2006.

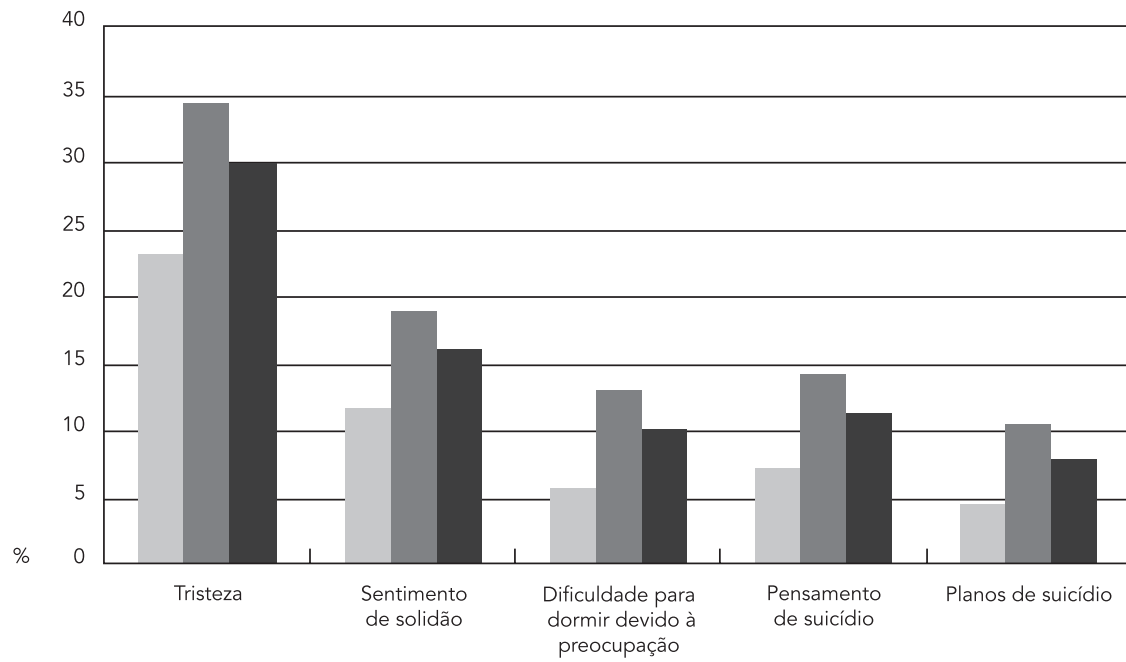


Análise de regressão logística bruta (odds ratio - OR e intervalo de 95\% de confiança - IC95\%) para regressão das variáveis independentes em relação aos indicadores de estresse psicossocial $(\mathrm{N}=4.210)$.

\begin{tabular}{|c|c|c|c|c|c|}
\hline Grupo/Variável & Tristeza & $\begin{array}{l}\text { Sentimento de } \\
\text { solidão }\end{array}$ & $\begin{array}{c}\text { Dificuldade para } \\
\text { dormir devido à } \\
\text { preocupação }\end{array}$ & $\begin{array}{c}\text { Pensamento em } \\
\text { suicídio }\end{array}$ & Planos de suicídio \\
\hline \multicolumn{6}{|l|}{ Rapazes } \\
\hline \multicolumn{6}{|c|}{ Exposição ao consumo de álcool } \\
\hline Não & 1,00 & 1,00 & 1,00 & 1,00 & 1,00 \\
\hline Sim & $1,11(0,87-1,43)$ & $0,84(0,59-1,20)$ & $0,99(0,64-1,52)$ & $1,60(1,11-2,29)$ & $1,32(0,81-2,14)$ \\
\hline \multicolumn{6}{|c|}{ Exposição ao tabagismo } \\
\hline Não & 1,00 & 1,00 & 1,00 & 1,00 & 1,00 \\
\hline Sim & $1,38(0,98-1,94)$ & $1,07(0,72-1,59)$ & $1,49(0,81-2,75)$ & $1,65(1,00-2,73)$ & $1,51(0,85-2,65)$ \\
\hline \multicolumn{6}{|c|}{ Exposição ao uso de drogas } \\
\hline Não & 1,00 & 1,00 & 1,00 & 1,00 & 1,00 \\
\hline $\operatorname{Sim}$ & $1,41(0,98-2,05)$ & $1,24(0,83-1,86)$ & $1,06(0,51-2,20)$ & $1,92(1,08-3,45)$ & $2,48(1,35-4,54)$ \\
\hline \multicolumn{6}{|l|}{ Moças } \\
\hline \multicolumn{6}{|c|}{ Exposição ao consumo de álcool } \\
\hline Não & 1,00 & 1,00 & 1,00 & 1,00 & 1,00 \\
\hline Sim & $1,58(1,34-1,86)$ & $1,37(1,09-1,76)$ & $1,40(1,06-1,85)$ & $1,92(1,51-2,45)$ & $1,82(1,37-2,42)$ \\
\hline \multicolumn{6}{|c|}{ Exposição ao tabagismo } \\
\hline Não & 1,00 & 1,00 & 1,00 & 1,00 & 1,00 \\
\hline $\operatorname{Sim}$ & $1,30(0,92-1,85)$ & $1,15(0,79-1,67)$ & $1,14(0,73-1,78)$ & $2,32(1,50-3,58)$ & $2,10(1,26-3,50)$ \\
\hline \multicolumn{6}{|c|}{ Exposição ao uso de drogas } \\
\hline Não & 1,00 & 1,00 & 1,00 & 1,00 & 1,00 \\
\hline Sim & $1,64(1,15-2,33)$ & $0,99(0,68-1,44)$ & $1,69(1,03-2,76)$ & $3,52(2,46-5,03)$ & $1,98(1,26-3,12)$ \\
\hline
\end{tabular}

ciado a pensamento de suicídio tanto em rapazes quanto em moças.

O presente estudo foi conduzido com um número relativamente grande de sujeitos, garantindo suficiente poder estatístico às análises. A abrangência do levantamento (estadual) e a seleção aleatória dos conglomerados possibilitam a extrapolação dos resultados para a populaçãoalvo do estudo (estudantes do Ensino Médio da rede pública estadual no Estado de Pernambuco). Os dados foram coletados com instrumento que foi previamente testado e apresentou boa consistência de medidas (reprodutibilidade), além disto, a aplicação foi realizada por equipe previamente treinada, fatores que podem ter minimizado a chance de viés de resposta.

No desenvolvimento do presente trabalho foi adotado delineamento transversal, assim deve-se interpretar com cautela o sentido das associações que foram identificadas devido à possibilidade de viés de causalidade reversa. Em adição, adolescentes podem omitir o consumo de bebidas alcoólicas, tabagismo e uso de drogas e, por conseguinte, conduzir à subestimativa da frequência de exposição a estas condutas de risco. Procurou-se contornar essa última limitação mediante a utilização de um questionário não identificado (anônimo) e pela garantia do sigilo das informações fornecidas. Além disso, os professores da escola não permaneciam na sala de aula durante a aplicação do questionário aos estudantes.

O tabagismo, o consumo de bebidas alcoólicas e o uso de drogas são condutas de risco à saúde que podem estar associados a outros indicadores de saúde mental e a transtornos mentais menores que não foram focalizados no presente estudo. Além disso, eventos estressantes, apoio social e violência, dentre outros, constituem potenciais fatores de confusão para as análises de associação que foram realizadas e que não puderam ser controlados, implicando a possibilidade de viés de confusão.

A amostra não abrangia adolescentes que estão matriculados na rede privada de ensino e aqueles que estão fora do sistema escolar, por isto, mesmo considerando que o estudo foi desenvolvido com uma amostra relativamente 
Análise de regressão logística (odds ratio - OR e intervalo de 95\% de confiança - IC95\%) para regressão das variáveis independentes em relação aos indicadores de estresse psicossocial, com ajustamento para as variáveis idade, morar com os pais, turno e demais fatores comportamentais de risco $(\mathrm{N}=4.210)$.

\begin{tabular}{|c|c|c|c|c|c|}
\hline Grupo/Variável & Tristeza & $\begin{array}{l}\text { Sentimento de } \\
\text { solidão }\end{array}$ & $\begin{array}{c}\text { Dificuldade para } \\
\text { dormir devido à } \\
\text { preocupação }\end{array}$ & $\begin{array}{c}\text { Pensamento em } \\
\text { suicídio }\end{array}$ & Planos de suicídio \\
\hline \multicolumn{6}{|l|}{ Rapazes } \\
\hline \multicolumn{6}{|c|}{ Exposição ao consumo de álcool } \\
\hline Não & 1,00 & 1,00 & 1,00 & 1,00 & 1,00 \\
\hline Sim & $1,00(0,76-1,30)$ & $0,78(0,54-1,14)$ & $0,93(0,59-1,47)$ & $1,55(1,06-2,26)$ & $1,23(0,68-2,21)$ \\
\hline \multicolumn{6}{|c|}{ Exposição ao tabagismo } \\
\hline Não & 1,00 & 1,00 & 1,00 & 1,00 & 1,00 \\
\hline $\operatorname{Sim}$ & $1,19(0,79-1,80)$ & $1,12(0,69-1,83)$ & $1,33(0,67-2,67)$ & $1,20(0,66-2,19)$ & $1,16(0,55-2,44)$ \\
\hline \multicolumn{6}{|c|}{ Exposição ao uso de drogas } \\
\hline Não & 1,00 & 1,00 & 1,00 & 1,00 & 1,00 \\
\hline Sim & $1,26(0,81-1,95)$ & $1,26(0,80-2,01)$ & $0,84(0,38-1,85)$ & $1,40(0,70-2,78)$ & $2,15(1,01-4,57)$ \\
\hline \multicolumn{6}{|l|}{ Moças } \\
\hline \multicolumn{6}{|c|}{ Exposição ao consumo de álcool } \\
\hline Não & 1,00 & 1,00 & 1,00 & 1,00 & 1,00 \\
\hline Sim & $1,48(1,25-1,76)$ & $1,36(1,04-1,78)$ & $1,29(0,97-1,72)$ & $1,62(1,24-2,12)$ & $1,68(1,23-2,31)$ \\
\hline \multicolumn{6}{|c|}{ Exposição ao tabagismo } \\
\hline Não & 1,00 & 1,00 & 1,00 & 1,00 & 1,00 \\
\hline Sim & $1,00(0,69-1,44)$ & $0,95(0,60-1,51)$ & $0,83(0,48-1,41)$ & $1,41(0,85-2,34)$ & $1,56(0,85-2,85)$ \\
\hline \multicolumn{6}{|c|}{ Exposição ao uso de drogas } \\
\hline Não & 1,00 & 1,00 & 1,00 & 1,00 & 1,00 \\
\hline $\operatorname{Sim}$ & $1,31(0,95-1,83)$ & $0,82(0,51-1,29)$ & $1,63(0,92-1,89)$ & $2,43(1,65-3,58)$ & $1,28(0,71-2,34)$ \\
\hline
\end{tabular}

grande, a generalização dos resultados deve ser limitada à população-alvo do estudo. Justifica-se, contudo, que a decisão de não incluir escolas do sistema privado de ensino no planejamento amostral foi decorrente da constatação de que mais de $85 \%$ dos adolescentes matriculados neste nível de ensino estão em escolas públicas estaduais. Há também evidências, tanto internacionais 16 quanto nacionais 19 , que indicam que a exposição ao consumo de bebidas alcoólicas, tabagismo e uso de drogas não é estatisticamente diferente entre os estudantes de escolas públicas e privadas.

No Município de Gravataí, Rio Grande do Sul, estudo realizado por Vieira et al. 20 evidenciou que o tabagismo estava associado ao sentimento de tristeza, solidão, dificuldade para dormir devido à preocupação e ideação suicida. Em pesquisa com adolescentes africanos, Peltzer 21 identificou resultados similares, apontando a existência de uma associação direta entre tabagismo e presença de indicadores de estresse psicossocial.
No presente estudo foram observados resultados divergentes em relação a essas duas investigações anteriormente mencionadas, mas que foram similares aos relatados por Rudatsikira et al. 18. Uma das possíveis explicações para essas discrepâncias foi o fato de que as análises realizadas no estudo de Vieira et al. 20 não foram conduzidas de modo a controlar potenciais fatores de confusão, e as análises de Peltzer 21 não foram estratificadas por sexo.

Em relação à associação entre exposição ao consumo de bebidas alcoólicas e indicadores de estresse psicossocial os resultados são igualmente divergentes. Enquanto Vieira et al. 20 e Peltzer 21 identificaram associações diretas entre esses fatores, no presente estudo, particularmente entre os rapazes, tais associações não foram observadas. Mais uma vez, a estratificação das análises por sexo e o controle de fatores intervenientes podem ser a principal explicação para as divergências entre os estudos.

Por outro lado, Vieira et al. 20 não identificaram associação entre uso de drogas na vida e 
sentimento de tristeza e dificuldade para dormir devido à preocupação. Resultado que converge para as evidências relatadas no presente estudo, no qual foi observada uma associação somente entre a exposição ao uso de drogas na vida com pensamento de suicídio, entre os rapazes, e com plano de suicídio, entre as moças.

No estudo de revisão conduzido por Kirby 22, os achados evidenciaram que na adolescência tanto o estresse psicossocial quanto o uso de álcool e drogas aumentam as chances de exposição ao comportamento sexual de risco. Similarmente, Lavan \& Johnson 23 verificaram que adolescentes expostos a estresse psicossocial têm maior chance de iniciação sexual precoce, não usar preservativo nas relações sexuais e gravidez indesejada. O estresse psicossocial está associado também ao baixo desempenho escolar 24 e envolvimento em atividades violentas e criminosas 25 .

$\mathrm{Na}$ última década, o suicídio representou uma importante causa de morte em adolescentes, particularmente em países desenvolvidos 16,26. Em pesquisa realizada em nove capitais brasileiras 27, verificou-se um aumento de 27,6\% entre 1979 e 1998 no número de mortes por causas externas em pessoas na faixa etária dos 15 aos 24 anos, sendo que o suicídio foi a sexta entre as causas de morte neste grupo. Se a situação brasileira ainda não é a mesma observada em países desenvolvidos, o aumento acelerado nas taxas de suicídio entre jovens sugere a necessidade de realização de mais estudos, particularmente no sentido de analisar como a medida do estresse psicossocial pode auxiliar o desenvolvimento de ações preventivas no campo da saúde mental.

Estudo realizado por Schilling et al. 28 evidenciou que o consumo de bebidas alcoólicas e, em especial, determinados padrões de consumo (isto é: consumo enquanto deprimido), tem associação com a tentativa de suicídio em adolescentes. Paralelamente, dados sobre o consumo de bebidas alcoólicas em populações jovens indicam que a exposição na vida e o consumo regular ocorrem em idades cada vez mais precoces 29 . Nesse cenário epidemiológico, a identificação de que a exposição ao consumo de bebidas alcoólicas é um fator associado ao pensamento e plano de suicídio neste grupo populacional pode fornecer evidências para intervenção e pesquisa.

Os achados desta investigação adicionam ao corpo de conhecimento disponível importantes evidências sobre a associação entre condutas de saúde e indicadores de estresse psicossocial em adolescentes. Dada a importância epidemiológica em populações jovens destes dois problemas de saúde pública, o uso de drogas e o suicídio, seria de fundamental importância o desenvolvimento de investigações que pudessem elucidar a relação causal entre estes fatores. Essas informações podem orientar o planejamento e a avaliação de ações sanitárias com melhor alocação dos recursos disponíveis. Um passo subsequente seria a investigação de intervenções para redução da exposição a condutas de risco à saúde.

\section{Resumo}

O objetivo deste artigo foi analisar a associação entre condutas de risco à saúde (tabagismo, consumo de bebidas alcoólicas e uso de drogas) e indicadores de estresse psicossocial em adolescentes estudantes do ensino médio. A pesquisa foi realizada com amostra constituída por 4.210 adolescentes estudantes de escolas públicas do Estado de Pernambuco, Brasil. O Global School-based Student Health Survey foi usado para coletar dados pessoais (demográficos e socioeconômicos) e comportamentais, e para obter medidas dos indicadores de estresse psicossocial (variáveis desfecho). Foram observadas prevalências de tristeza, sentimento de solidão, pensamento de suicídio, dificuldade para dormir devido à preocupação e planos de suicídio. Tabagismo, consumo de bebidas alcoólicas e uso de drogas foi relatado, respectivamente, por 7,7\%, 30,3\% e 6,9\%. As prevalências de indicadores de estresse psicossocial foram maiores entre as moças, e as prevalências de exposição a condutas de risco à saúde foram maiores entre os rapazes. Concluiu-se com o estudo que o uso de drogas está diretamente associado ao pensamento e plano de suicídio e, entre as moças, o consumo de bebidas alcoólicas foi um fator associado ao estresse psicossocial.

Estresse Psicológico; Suicídio; Adolescente; Conduta de Saúde 


\section{Colaboradores}

P. D. Carvalho participou da análise de dados, discussão e interpretação de resultados, e análise final do artigo. M. V. G. Barros, R. A. Lima, C. M. Santos e E. N. Mélo colaboraram na análise de dados e revisão final do artigo.

\section{Agradecimentos}

Ao Conselho Nacional de Desenvolvimento Científico e Tecnológico (CNPq) pela concessão de auxílio financeiro a projeto de pesquisa (processo 486023/2006-0) e bolsa de produtividade em pesquisa (processo 307415/2010-4). Fundação de Amparo à Ciência e Tecnologia do Estado de Pernambuco (FACEPE) e Coordenação de Aperfeiçoamento de Pessoal de Nível Superior (CAPES) pela concessão de bolsas de estudo e recursos de custeio para o desenvolvimento do projeto.

\section{Referências}

1. Ellison RC. Commentary 3. Early interventions: the population approach. In: Cheung LWY, Richmond JB, editors. Child, health, nutrition and physical activity. Champaign: Human Kinetics; 1995; p. 293-7.

2. Aarnio M, Winter T, Peltonen J, Kujala UM, Kaprio J. Stability of leisure-time physical activity during adolescence--a longitudinal study among 16-, 17and 18-year-old Finnish youth. Scand J Med Sci Sports 2002; 12:179-85.

3. Malyutina S, Bobak M, Kurilovitch S, Gafarov V, Simonova G, Nikitin Y, et al. Relation between heavy and binge drinking and all-cause and cardiovascular mortality in Novosibirsk, Russia: a prospective cohort study. Lancet 2002; 360:1448-54.

4. Flouris AD, Faught BE, Klentrou P. Cardiovascular disease risk in adolescent smokers: evidence of a "smoker lifestyle". J Child Health Care 2008; 12:221-31.

5. Baumgartner JN, Waszak GC, Tucker H, Wedderburn M. The influence of early sexual debut and sexual violence on adolescent pregnancy: a matched case-control study in Jamaica. Int Perspect Sex Reprod Health 2009; 35:21-8.

6. Rivera-Rivera L, Allen-Leigh B, Rodriguez-Ortega G, Chavez-Ayala R, Lazcano-Ponce E. Prevalence and correlates of adolescent dating violence: baseline study of a cohort of 7,960 male and female Mexican public school students. Prev Med 2007; 44:477-84.
7. Nduna M, Jewkes RK, Dunkle KL, Shai NP, Colman I. Associations between depressive symptoms, sexual behaviour and relationship characteristics: a prospective cohort study of young women and men in the Eastern Cape, South Africa. J Int AIDS Soc 2010; 13:44.

8. Eaton DK, Kann L, Kinchen S, Shanklin S, Ross J, Hawkins J, et al. Youth risk behavior surveillance United States, 2009. MMWR Surveill Summ 2010; 59:1-142.

9. Niclasen B, Schnohr C. Has the curve been broken? Trends between 1994 and 2006 in smoking and alcohol use among Greenlandic school children. Int J Circumpolar Health 2008; 67:299-307.

10. Andrade SM, Soares DA, Braga GP, Moreira JH, Botelho FMN. Comportamentos de risco para acidentes de trânsito: um inquérito entre estudantes de medicina na região sul do Brasil. Rev Assoc Med Bras 2003; 49:439-44.

11. Coordenação Geral de Doenças e Agravos Não Transmissíveis, Secretaria de Vigilância em Saúde, Ministério da Saúde. Pesquisa Nacional de Saúde do Escolar - PeNSE. Brasília: Ministério da Saúde; 2006.

12. Farias Júnior JC, Nahas MV, Barros MVG, Loch MR, Oliveira ESA, Bem MFL, et al. Comportamentos de risco à saúde em adolescentes no Sul do Brasil: prevalência e fatores associados. Rev Panam Salud Pública 2009; 25:344-52. 
13. Brener ND, Collins JL. Co-occurrence of healthrisk behaviors among adolescents in the United States. J Adolesc Health 1998; 22:209-13.

14. Sychareun V, Thomsen S, Faxelid E. Concurrent multiple health risk behaviors among adolescents in Luangnamtha province, Lao PDR. BMC Public Health 2011; 11:36.

15. Harling M, Strehmel P, Schablon A, Nienhaus A. Psychosocial stress, demoralization and the consumption of tobacco, alcohol and medical drugs by veterinarians. J Occup Med Toxicol 2009; 4:4.

16. Mahfoud ZR, Afifi RA, Haddad PH, Dejong J. Prevalence and determinants of suicide idetion among Lebanese adolescents: results of the GSHS Lebanon 2005. J Adolesc 2010; 34:379-84.

17. Page RM, Hall C. Psychosocial distress and alcohol use as factors in adolescent sexual behavior among sub-Saharan African adolescents. J Sch Health 2009; 79:369-79.

18. Rudatsikira E, Muula AS, Siziya S, Twa-Twa J. Suicidal ideation and associated factors among school-going adolescents in rural Uganda. BMC Psychiatry 2007; 7:67.

19. Sanceverino SL, Abreu JLC. Aspectos epidemiológicos do uso de drogas entre estudantes do ensino médio no Município de Palhoça 2003. Ciênc Saúde Coletiva 2004; 9:1047-56.

20. Vieira PC, Aerts DRGC, Freddo SL, Bittencourt A, Monteiro L. Uso de álcool, tabaco e outras drogas por adolescentes escolares em município do Sul do Brasil. Cad. Saúde Pública 2008; 24:2487-98.

21. Peltzer K. Prevalence and correlates of substance use among school children in six African countries. Int J Psychol 2009; 44:378-86.
22. Kirby D. Antecedents of adolescent initiation of sex, contraceptive use, and pregnancy. Am J Health Behav 2002; 26:473-85.

23. Lavan H, Johnson JG. The association between axis I and II psychiatric symptoms and high-risk sexual behavior during adolescence. J Pers Disord 2002; 16:73-94.

24. Rothon C, Head J, Clark C, Klineberg E, Cattell V, Stansfeld S. The impact of psychological distress on the educational achievement of adolescents at the end of compulsory education. Soc Psychiatry Psychiatr Epidemiol 2009; 44:421-7.

25. Ritakallio M, Haltiala-Heino R, Kivivouri J, Rimpela M. Delinquent behaviour and depression in middle adolescence: A Finnish community sample. J Adolesc 2005; 28:155-9.

26. Gibbons RD, Brown CH, Hur K, Marcus SM, Bhaumik DK, Erkens JA, et al. Early evidence on the effects of regulators' suicidality warnings on SSRI prescriptions and suicide in children and adolescents. Am J Psychiatry 2007; 164:1356-63.

27. Souza ER, Minayo MCS, Malaquias JV. Suicide among young people in selected Brazilian State capitals. Cad Saúde Pública 2002; 18:673-83.

28. Schilling EA, Aseltine RH, Glanovsky JL, James A, Jacobs D. Adolescent alcohol use, suicidal ideation, and suicide attempts. J Adolesc Health 2009; 44:335-41.

29. Soldera M, Dalgalarrondo P, Corrêa Filho HR, Silva CAM. Uso de drogas psicotrópicas por estudantes: prevalência e fatores associados. Rev Saúde Pública $2004 ; 38: 277-83$.

Recebido em 01/Fev/2011

Versão final reapresentada em 02/Ago/2011

Aprovado em 22/Ago/2011 